\title{
Modelo computacional 3D para estimar variables eléctricas inducidas en un cultivo in vitro estimulado por campos magnéticos de baja frecuencia
}

\author{
María E. Moncada ${ }^{\S *}$, Carlos R. Pinedo**, William D. Criollo ***, Jose O. Gutiérrez***, \\ Hector Cadavid** \\ *Instituto Tecnológico Metropolitano, Institución Universitaria; Centro de Investigación. \\ ** Universidad del Valle, Facultad de Ingeniería, Escuela de Ingeniería Eléctrica y Electrónica. \\ ***Universidad del Valle, Facultad de Salud, Escuela de Farmacología. \\ §mariamoncada@itm.edu.co \\ (Recibido: Noviembre 19 de 2009 - Aceptado: Noviembre 05 de 2010)
}

\section{Resumen}

Los modelos computacionales son un área en continuo desarrollo e investigación que se usan como apoyo a los procedimientos experimentales. Estos representan una herramienta fundamental para definir variables cada vez más específicas a aplicarse reduciendo tiempo de investigación y dinero. En este estudio se desarrolló un modelo computacional 3D de células de fibroblastos y osteoblastos humanas estimulados por campos magnéticos. El modelo se creó a partir del esquema experimental real formado por la fuente (Bobina Helmholtz), una placa Falcon ${ }^{\mathrm{TM}}$ de 96 pozos y el material celular. La resistencia eléctrica para las células se midió en cada material biológico y en el modelo se asignó la resistividad. La densidad de flujo magnético aplicada fue de 1.0 y $1.5 \mathrm{mT}$ y frecuencias entre 15 y $105 \mathrm{~Hz}$. Las variables evaluadas fueron el campo eléctrico, la densidad de corriente y el calentamiento eléctrico. Se observó un crecimiento exponencial de las señales inducidas con la frecuencia y la densidad de flujo magnético generado, más significativo para el primer caso.

Palabras Claves: Estimulación celular, Modelo computacional, Densidad de flujo magnético, Fibroblastos, Osteoblastos.

\section{D computer model to estimate induced electrical parameters in an in-vitro culture stimulated by low-frequency magnetic fields}

\begin{abstract}
Computational models are a continuous topic in research and development, which is used to support experimental procedures. These represent a fundamental tool to define increasingly specific variables to be applied by reducing costs and research time. In this study, a 3D computer model of human fibroblast and osteoblast cells stimulated by magnetic fields was developed. The model was created from the actual experimental scheme. It was constituted by the source (a Helmholtz coil), a FalconTM 96-well cell culture plate, and the cellular material. The electrical resistivity property of cells was measured in each material and the electrical resistivity property was assigned to the computer model. The applied magnetic flux density was $1 \mathrm{mT}$ and $1,5 \mathrm{mT}$ with frequencies between $15 \mathrm{~Hz}$ and $105 \mathrm{~Hz}$. Electric field, current density, and electric heating were the variables evaluated. It was observed an exponential growth of induced signals with frequency and generated magnetic field level, this being more significant for the frequency.
\end{abstract}

Keywords: Cellular stimulation, Computational model, Magnetic field, Fibroblast, Osteoblast 


\section{Introducción}

El uso de la terapia con campos electromagnéticos (CEM), se sigue presentando como una alternativa a tratamientos farmacológicos sin efectos secundarios o tóxicos [FDA]. La evidencia muestra la aplicación de CEM para diferentes tratamientos tales como desordenes ortopédicos (Bjordal et al, 2003; Ferguson et al, 2005; Ojingwa \& Isseroff, 2003; Sluka \& Walsh, 2003), en piel (Lee et al, 1993; Gentzkow, 1993), tipos de cáncer (Gothelf et al, 2003), dolores agudos, entre otras.

Diferentes estudios de cultivos celulares de osteoblastos in vitro estimulados con CEM de extremada baja frecuencia han mostrado proliferación; incremento en la concentración del factor IGF-II, afectando el metabolismo de cartílago articular y previniendo la perdida de hueso (Fitzsimmons et al, 1995) y efectos en el transporte de iones de calcio (Fitzsimmons et al, 1994). Algunos experimentos sobre fibroblastos encontraron respuesta en la microcirculación y estimulación de proliferación y diferenciación (Katsir et al, 1998). Se encontró además refuerzo en la síntesis de DNA con CEM para frecuencias entre $15 \mathrm{~Hz}$ y $4 \mathrm{kHz}$.

Con lo descrito, el desarrollo de modelos computacionales que permitan explorar el efecto de la densidad de flujo magnético (denominado en este artículo como campos magnéticos) en los tejidos celulares, puede llegar a ser un apoyo importante a los estudios de evaluación in vitro, ahorrando con esto tiempo de investigación y dinero.

En las instituciones relacionadas se han venido trabajando en el desarrollo de modelos computacionales (Moncada et al, 2007; 2008) que esperan realimentarse con los resultados de los estudios experimentales y clínicos que se adelantan, para llegar a convertirlos en una herramienta de apoyo a estas aplicaciones.

En este estudio se creó un modelo computacional $3 \mathrm{D}$ para representar un sistema de cultivo celular in vitro estimulado por campos magnéticos de baja frecuencia. El modelo computacional se desarrolló en el programa Ansys, el cual se basa en la solución a las ecuaciones de Maxwell utilizando el método de elementos finitos para el análisis electromagnético. Al modelo computacional lo componen una bobina Helmholtz como fuente de estimulación magnética, una caja representando la placa Falcon de cultivo celular y cilindros 3D representando el material celular de fibroblastos y osteoblastos humanos. La propiedad asignada a cada parte del modelo fue la resistividad y el campo magnético generado fue sinusoidal con magnitud 1.0 y $1.5 \mathrm{mT}$ y frecuencia entre 15 y 105 $\mathrm{Hz}$. Las señales eléctricas inducidas estudiadas fueron el campo eléctrico, la densidad de corriente y el calentamiento eléctrico.

En el modelo computacional, se observó que las señales eléctricas inducidas presentaron crecimiento exponencial al aumentar la señal generada en campo magnético y en frecuencia, y que dicho cambio fue más significativo para el segundo caso.

\section{Metodología}

\subsection{Obtención de las muestras celulares}

Dos tipos de células fueron representadas en el modelo computacional: osteoblastos-cultivo primario tomadas de sobrantes de material hemático de la medula ósea, con su respectivo protocolo de inclusión y aceptación de los pacientes a participar en el estudio y fibroblastos de piel humana normal que se encontraban almacenados en termos con nitrógeno líquido de la Asociación IN-VITRO, de la Universidad del Valle.

\section{Osteobastos-cultivo primario}

Las muestras se tomaron de la sangre que compone la medula ósea (con todas sus células) de la cual fueron aislados los osteoblastos. El tejido se trituró y posteriormente se transfirió al medio para crecimiento de osteoblastos. Después del tercero o cuarto día in vitro (3-4 DIV) se realizó el cambio de medio total. El cultivo se observó cada día hasta la visualización de la formación de agregados celulares. Cuando se alcanzó el porcentaje óptimo de confluencia, se procedió a desprender la monocapa. Al obtener desprendimiento de las células, se agregó un volumen igual de medio de crecimiento para inhibir la acción de la enzima. 
Los fibroblastos se almacenaron en termos con nitrógeno líquido de la Asociación IN-VITRO, de la Universidad del Valle. Previamente caracterizados se trabajaron en pase 5 de crecimiento. Para los análisis se utilizaron FPHN en pase 6, previa descongelación de las células.

\section{Procedimiento de descongelación}

El procedimiento de descongelación fue igual para las células de osteoblastos y fibroblastos. El material se procesó hasta obtener el aislamiento celular, descongelado y recuperado por centrifugación. Posteriormente se sembraron con medio de crecimiento el cual se cambió después del día 2 o 3 In vitro (2-3 DIV). Terminado el tiempo de incubación las células se observaron al microscopio para verificación del desprendimiento celular; cuando éste ocurrió se adicionó medio de crecimiento para inhibir la acción de la tripsina.

\section{Grupos de radiación}

Las células se depositaron en 16 de los 96 pozos de la placa con el fin de garantizar su exposición en un campo magnético homogéneo (Figura 1). Los grupos de radiación evaluados se establecieron variando la magnitud de campo magnético en $1.0 \mathrm{y}$ $1.5 \mathrm{mT}$; para frecuencia entre 15 y $105 \mathrm{~Hz}$ y diferentes tiempos de estimulación. Para cada grupo de radiación se contó con su grupo control.

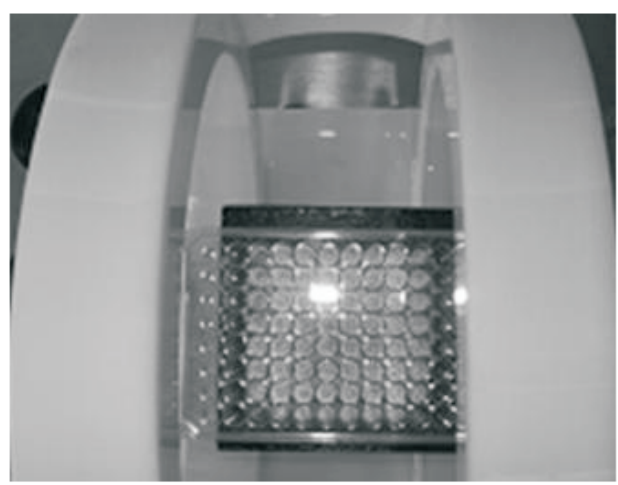

(a)

\subsection{Equipo estimulador}

El equipo de estimulación magnética se diseñó y se construyó en los laboratorios de la Universidad del Valle. Está compuesto por un amplificador de potencia que permite entregar voltaje y corriente variables en el tiempo, cuenta con protecciones de sobre voltaje y corto circuito y está conectado a una bobina Helmholtz para la generación del campo magnético. El campo magnético generado es unidireccional sobre el eje z con magnitud entre $0.5 \mathrm{mT}$ y $1.5 \mathrm{mT}$ y frecuencia entre $15 \mathrm{~Hz}$ y $105 \mathrm{~Hz}$. Las características de la fuente se establecieron de acuerdo a la geometría de las bobinas (pues fue diseñada para estos valores de carga). En las pruebas técnicas se verificó el campo magnético generado ante la variación de la corriente y la frecuencia, también se realizaron pruebas de reproducibilidad para estas dos variables. Las pruebas fueron apoyadas por el Gaussímetro Serie 5100 y las señales se observaron y se registraron en un osciloscopio digital.

\subsection{Propiedades eléctricas}

La resistencia eléctrica se midió para los dos materiales celulares. El equipo utilizado fue el Fluke 87 y previo a la medición sus puntas se esterilizaron a gas por 12 horas. Para no interferir o contaminar las células radiadas. La medición de la resistencia se realizó en un pozo de $3.5 \mathrm{~cm}^{2}$ cinco días después de sembradas las células para

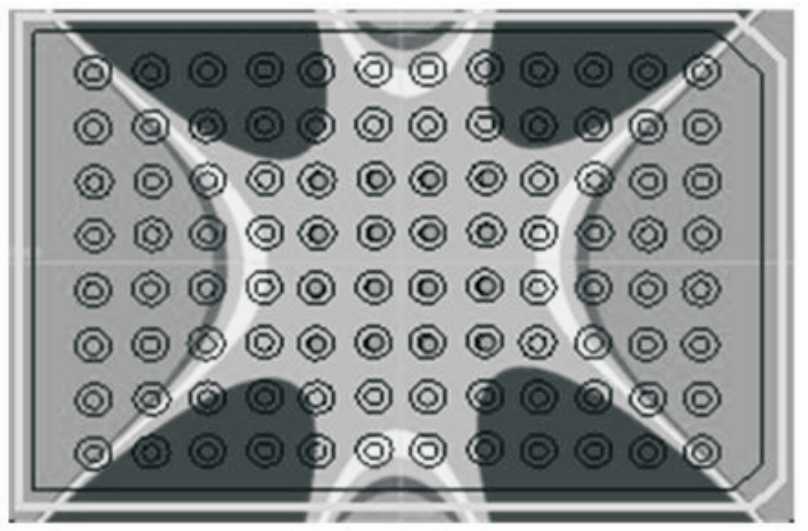

(b)

Figura 1. a) Células ubicadas en el centro de las bobinas; b) representación computacional del campo magnético en el que fueron ubicadas las células. 


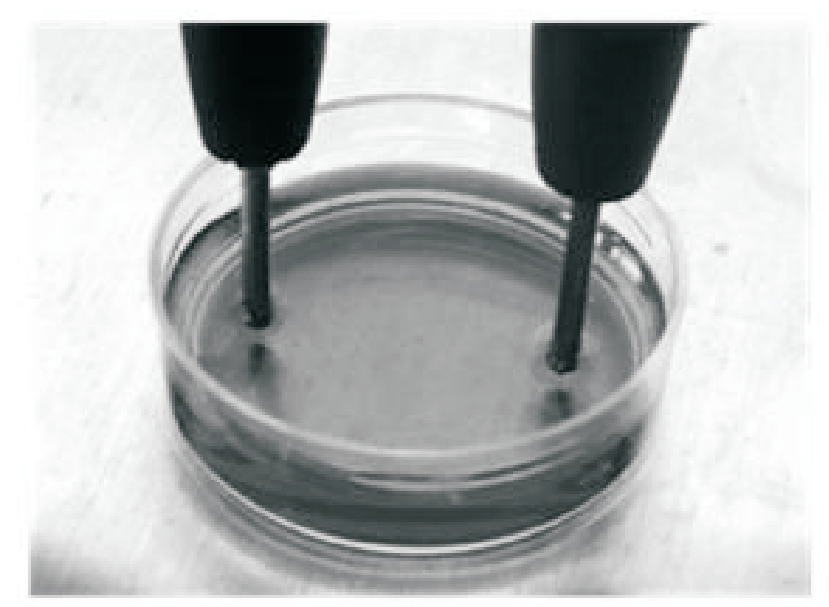

Figura 2. Esquema de medición de la resistencia del material biológico en un pozo de $3.5 \mathrm{~cm}^{2}$ sobre material no radiado.

lograr la confluencia de la monocapa y sobre material no radiado, como se ilustra en la Figura 2. Dada la inhomogeidad del material celular, se realizaron, para cada material, cinco mediciones instantáneas y el promedio entre ellas fue el asignado al modelo. Luego de cada dato los electrodos se sumergieron en solución salina $\mathrm{NaCl}$ al $0.9 \%$ con el fin de despolarizar los electrodos antes de una nueva medición (Cromwell, et al 1980). Es de aclarar que no fue realizado un estudio riguroso en la medición de las propiedades $\mathrm{y}$ este es uno de los puntos mejorados en un estudio actual.

La resistividad del medio se encontró a partir de la geometría de la celda y de los electrodos de medición, utilizando la Ec. (1).

$$
\rho=\frac{A \times R}{l}
$$

Con $\rho$, resistividad; $A$, área efectiva del electrodo; $R$, resistencia y $l$, separación entre electrodos. Con la forma cónica de los electrodos, el área efectiva se encontró a partir de la Ec. (2).

$$
A_{t}=\pi \mathrm{rg}+\pi \mathrm{r}^{2}
$$

Con $A t$, área total efectiva; $r$ : radio del círculo; $g$, lado del cono.
Las resistividades eléctricas asignadas a cada material en el modelo computacional fueron: para las bobinas el cobre, para la placa Falcon el acrílico y para el material celular los valores de resistividad eléctrica obtenidos

\subsection{Modelo computacional arreglo bobina- células}

\section{Geometría}

La geometría del sistema completo bobinacélulas se construyó en 3D y está conformada por cuatro partes: a) la bobina Helmholtz circular representando la fuente, b) la placa Falcon, c) cilindros macizos representando el contenido celular y d) un cilindro suficientemente grande que contiene las geometrías anteriores al cual es asignado el potencial cero. En la primera parte del modelo se construyó y se simuló sólo la bobina Helmholtz (con las mismas características del equipo construido), en este se evaluaron el área y la magnitud del campo magnético homogéneo generado y los datos obtenidos se compararon con los valores arrojados por las pruebas técnicas del equipo (Figura 6). El campo magnético se generó unidireccional en el eje $z$. La Figura 3 ilustra las líneas y los volúmenes creados. Por limitación computacional, se pudo construir solo 36 de los 96 pozos de la placa Falcon, de los cuales 16 corresponden a los pozos con contenido celular. 


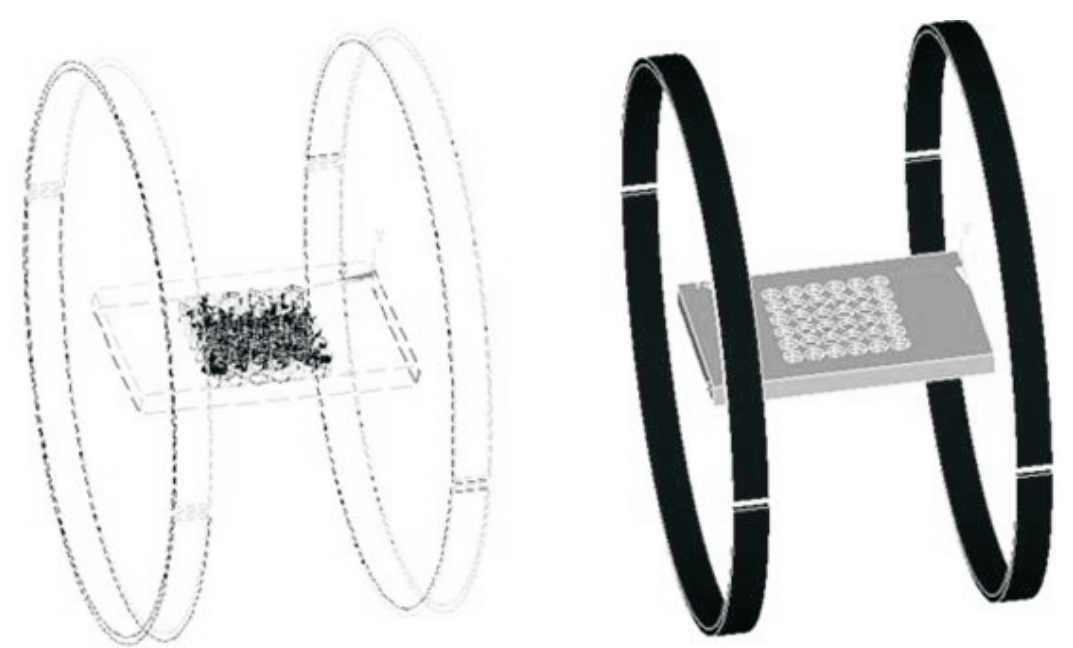

Figura 3. Representación geométrica computacional del arreglo bobina-células.

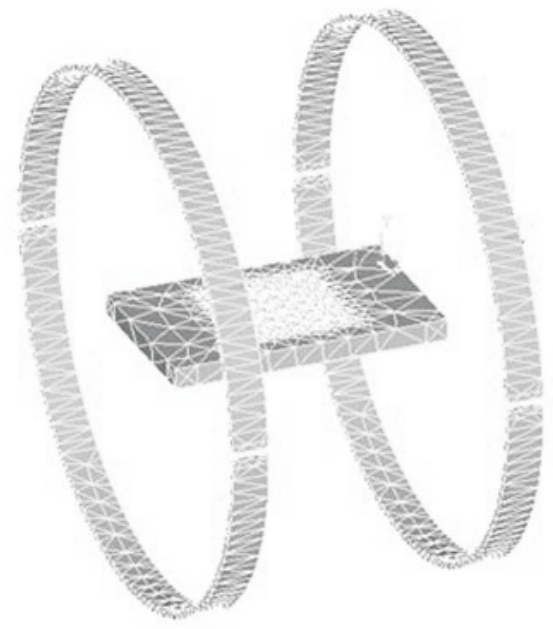

Figura 4. Arreglo bobina-células enmallados para el análisis electromagnético

\section{Propiedades}

Para el análisis electromagnético se utilizaron las propiedades de resistividad eléctrica y permeabilidad magnética, las propiedades fueron consideradas isotrópicas, no fueron considerados valores de permitividad (por trabajar en condiciones cuasi-estáticas) y el valor de permeabilidad relativa usada fue de 1 .

\section{Análisis electromagnético}

Una vez construida la geometría y asignadas las propiedades, se realizó la caracterización de las condiciones de carga y mallado para el análisis electromagnético (el programa Ansys utiliza el método de los elementos finitos para la solución de las ecuaciones de Maxwell). La fuente generadora (bobina Helmholtz) se caracterizó como material conductor sólido alimentado por voltaje (a partir de una diferencia de potencial) con dos grados de libertad (voltaje y vector potencial magnético) y sin considerar efectos de corrientes de eddy. Las señales de la fuente fueron las mismas utilizadas en el desarrollo experimental (campo magnético de 1.0 y $1.5 \mathrm{mT}$ y frecuencias entre 15 y $105 \mathrm{~Hz}$ ). El arreglo bobina-células mallado puede observarse en la Figura 4. El modelo computacional permite evaluar diferentes condiciones de carga y tamaños de las bobinas. 


\section{Resultados y discusión}

\subsection{Propiedades eléctricas}

La Tabla 1, resume los valores de resistividad y conductividad (Ec. 1 y 2) encontrados para los dos tipos de células de fibroblastos y osteoblastos, asignadas como isotrópicas en el modelo computacional.

\subsection{Bobinas}

La Figura 5 ilustra la geometría real y computacional 3D de la bobina Helmholtz creadas. La Figura 6 compara los valores medidos y simulados para una bobina Helmholtz de 12.5 $\mathrm{cm}$ de radio en corriente vs. el campo magnético generado. El error máximo encontrado entre los valores medidos y simulados fue de $4.4 \%$. Para ambos arreglos de bobina (construido y simulado) se encontró estabilidad en la señal de la fuente la cual no presentó cambios en el campo magnético generado ante las variaciones de la frecuencia.

\subsection{Arreglo bobina-células}

La Figura 7, ilustra los modelos 3D de la bobina Helmholtz, la placa Falcon y los cilindros representando el material celular.

El campo magnético, en el modelo computacional, fue unidireccional al eje $z$ (igual que la condición real de estimulación). Las variables eléctricas inducidas evaluadas fueron el campo magnético total, la densidad de corriente para los ejes $x, y$ y $z$, el campo eléctrico para los ejes $x, y$ y $z$ y el calentamiento eléctrico total.

La Figura 8 muestra el área de campo magnético homogéneo y en la Figura 9, la distribución de la densidad de corriente en los volúmenes que representaron el material celular.

Tabla 1. Valores de conductividad y resistividad para los materiales biológicos del estudio

\begin{tabular}{lcc}
\hline \multicolumn{1}{c}{ Material } & $\sigma_{X, Y, Z}(\mathrm{~S} / \mathrm{m})$ & $\rho_{X, Y, Z}(\Omega . \mathrm{m})$ \\
\hline Fibroblastos de piel humana normal & $7.719 \mathrm{e}-4$ & 1295.5 \\
Líneas celulares de osteoblastos & $5.146 \mathrm{e}-4$ & 1943.25 \\
\hline
\end{tabular}

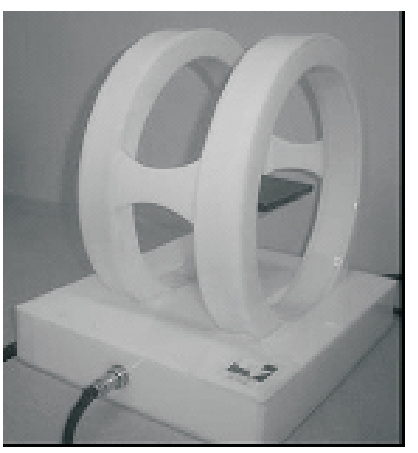

(a)

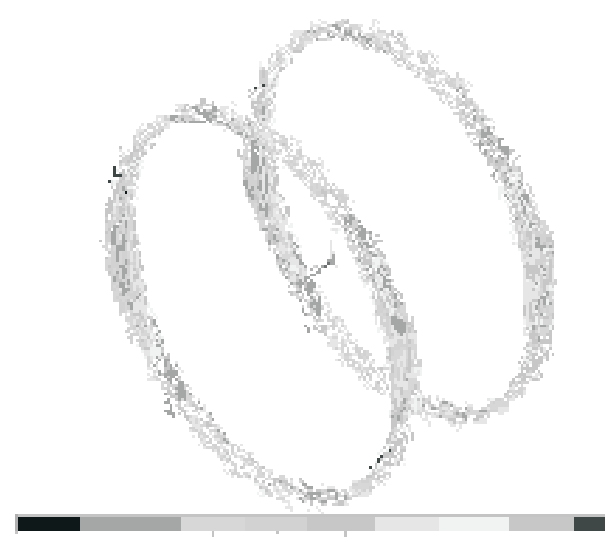

(b)

Figura 5 Geometría de las bobinas a) real, b) computacional 3D. 


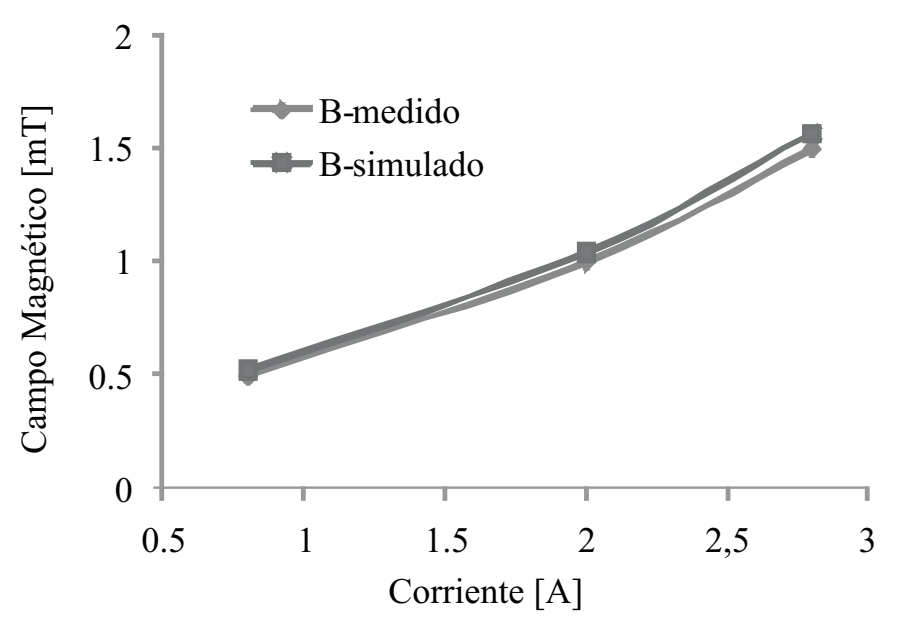

Figura 6. Corriente vs. campo magnético para valores medido y simulados en el programa Ansys.

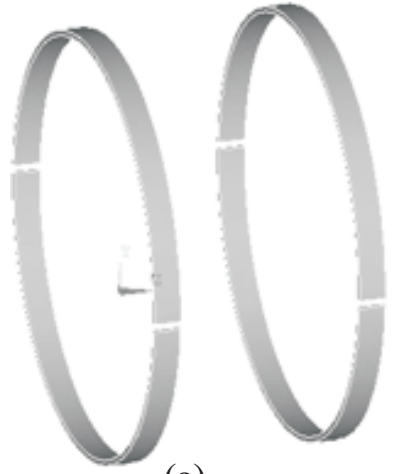

(a)

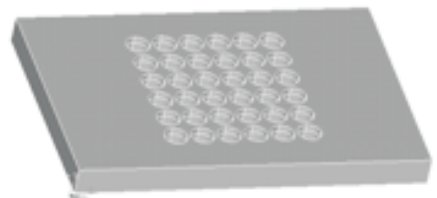

(b)

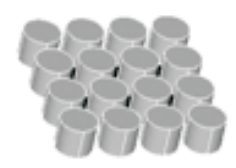

(c)

Figura 7. Geometría computacional 3D de los elementos (a) bobinas; (b) placa Falcon; (C) cilindros representando el contenido celular.

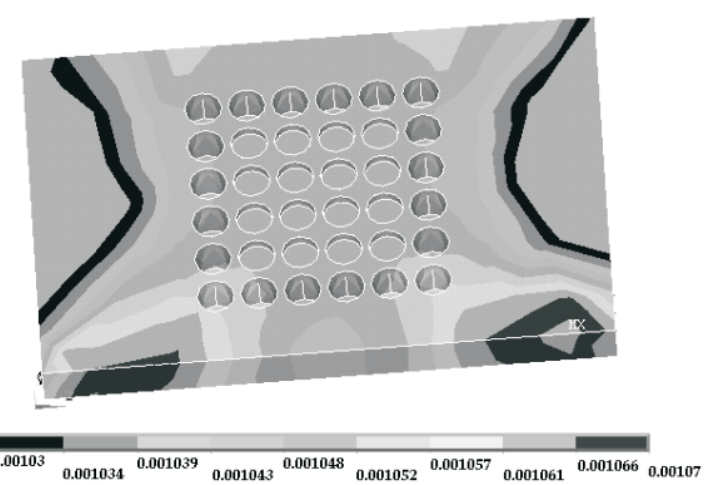

Figura 8. Campo magnético inducido sobre el modelo de la placa y el material biológico. 
La Figura 10 presenta para las células de fibroblastos, a) la densidad de corriente inducida vs. frecuencia sobre los ejes $x, y$ y $z$ y b) el campo eléctrico inducido vs. frecuencia sobre los ejes $x, y$ y $z$. El comportamiento fue similar para las células de osteoblastos.
La Tabla 2 presenta el porcentaje de crecimiento en cuanto a la densidad de corriente y campo eléctrico inducido (componente $J y$ ) en el modelo de fibroblastos y osteoblastos ante el cambio del campo magnético y la frecuencia de la fuente.

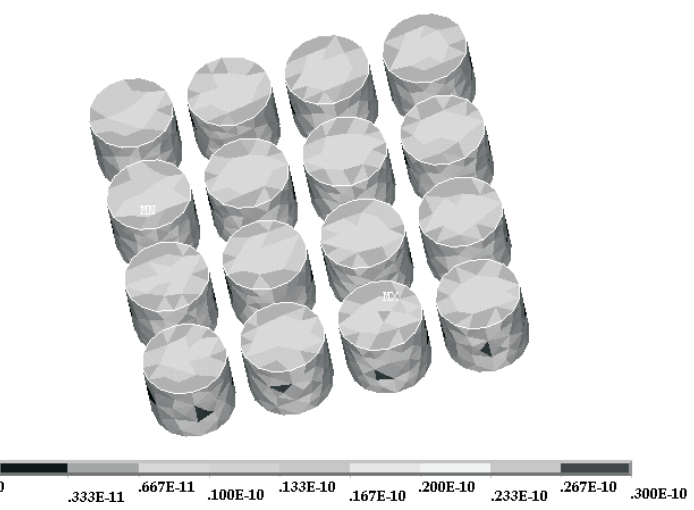

Figura 9. Distribución de la densidad de corriente en los volúmenes representando el material celular.

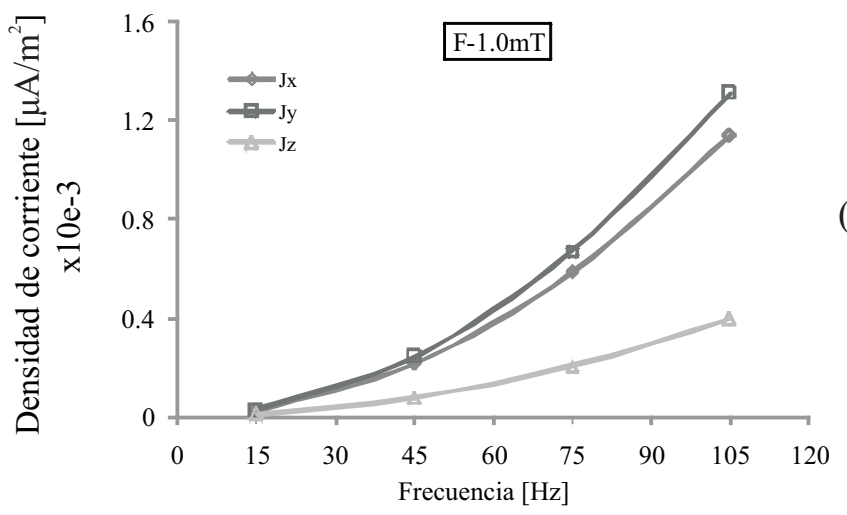

(a)

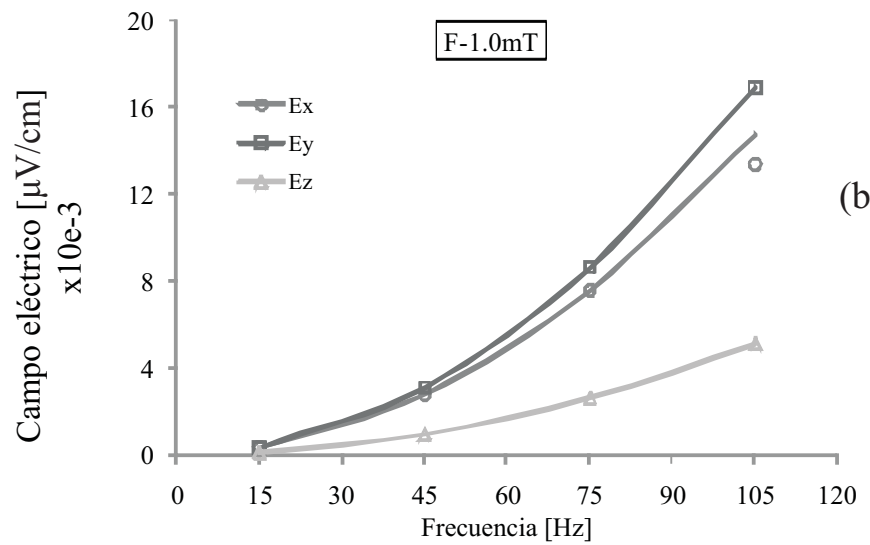

Figura 10. a) Densidad de corriente inducida vs. frecuencia para los ejes $x$, $y y z$, b) Campo eléctrico inducido vs. frecuencia para los ejes $x, y$ y $z$. 
Tabla 2. Porcentaje de crecimiento en la densidad de corriente y campo eléctrico inducido (componente $J_{\nu}$ ) en el modelo de fibroblastos y osteoblastos ante el cambio del campo magnético y la frecuencia de la fuente.

\begin{tabular}{lrrrr}
\hline & \multicolumn{2}{c}{ Densidad de corriente } & \multicolumn{2}{c}{ Campo eléctrico } \\
\hline Fibroblastos & & & & \\
$1.0-1.5 \mathrm{mT}^{(1)}$ & 40.43 & 40.35 & 39.78 & 39.69 \\
$15 \mathrm{~Hz}-105 \mathrm{~Hz}^{(2)}$ & 4751.06 & 4748.48 & 4769.89 & 4767.02 \\
& & & & \\
Osteoblastos & 40.13 & 40.20 & 40.22 & 40.23 \\
$1.0-1.5 \mathrm{mT}^{(1)}$ & 4759.87 & 4763.64 & 4760.34 & 4760.56 \\
$15 \mathrm{~Hz}-105 \mathrm{~Hz}^{(2)}$ & & & \\
\hline
\end{tabular}

(1) Para las frecuencias fijas de $15 \mathrm{~Hz}$ y 105Hz; (2) Para el campo magnético fijo de $1.0 \mathrm{mT}$ y $1.5 \mathrm{mT}$.

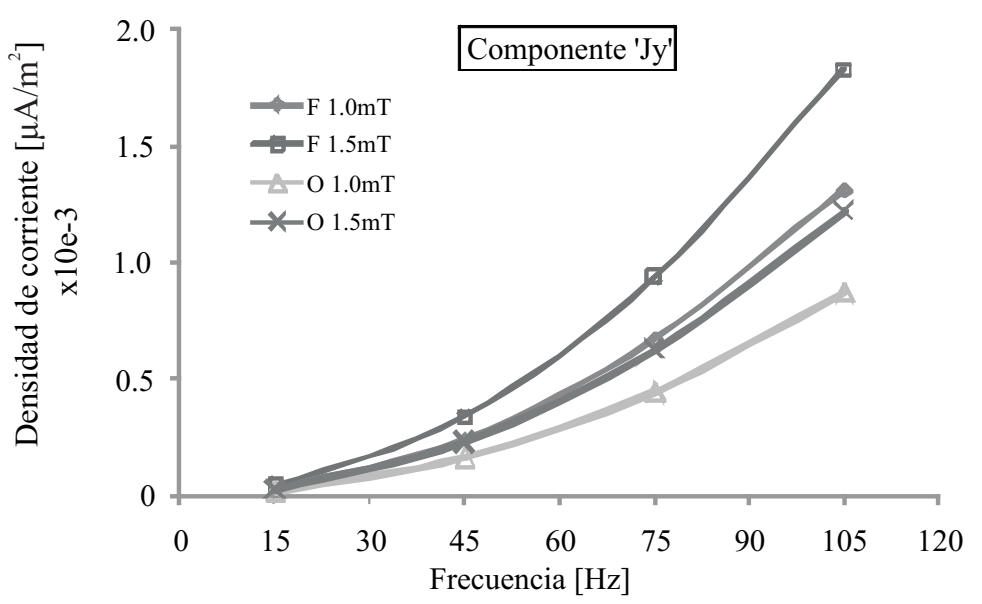

Figura 11. Densidad de corriente inducida vs. Frecuencia componente y, en fibroblastos y osteoblastos a $1.0 \mathrm{~m}$ T y $1.5 \mathrm{mT}$.

Para ambos materiales se encontró: 1) al aumentar la frecuencia de $15 \mathrm{~Hz}$ a $105 \mathrm{~Hz}$, la densidad de corriente inducida aumentó en 50 veces para cada componente, los valores superiores fueron encontrados para la componente $J_{y}$ (alto del cilindro celular) y los inferiores para la componente $J_{z}$ (dirección del campo magnético generado); 2) el campo eléctrico, al igual que la densidad de corriente creció exponencialmente con la frecuencia en las mismas 50 veces al pasar de $15 \mathrm{~Hz}$ a $105 \mathrm{~Hz}$; 3) Los mayores valores fueron encontrados para la componente $E_{y}$ e inferior para la componente $E_{z}$; 4) La densidad de corriente y el campo eléctrico, fueron superiores en la componente $y$ la cual fue 1,2 veces la componente $x$ y 3 veces la componente $z$; 5) Los valores de densidad de corriente y campo eléctrico inducido fueron superiores para la señal de $1.5 \mathrm{mT}$ en 1.5 veces, sobre la señal de $1,0 \mathrm{mT}$ para todas las frecuencias; 6) $\mathrm{Al}$ aumentar la frecuencia de $15 \mathrm{~Hz}$ a $105 \mathrm{~Hz}$, la señal creció hasta 50 veces para ambos valores de campo magnético 1.0 y $1.5 \mathrm{mT}$; 7) la magnitud de la densidad de corriente y el campo eléctrico inducidos, fueron superiores para los fibroblastos (del orden de $10^{-9}$ ) sobre los osteoblastos (del orden de $10^{-10}$ ); 8) el cambio en la densidad de corriente y el campo eléctrico fue más significativo ante el aumento de la frecuencia (de $15 \mathrm{a}$ a105 Hz) que ante el aumento del campo magnético generado $(1.0-1.5 \mathrm{mT})$.

La Figura 11 muestran la densidad de corriente inducida $J_{y}$ vs. frecuencia para los dos tipos de células y las dos magnitudes de campo magnético generado. Las Figuras 12 y 13 presentan el campo eléctrico inducido vs. frecuencia y el calentamiento eléctrico vs. Frecuencia respectivamente, para las mismas condiciones. 


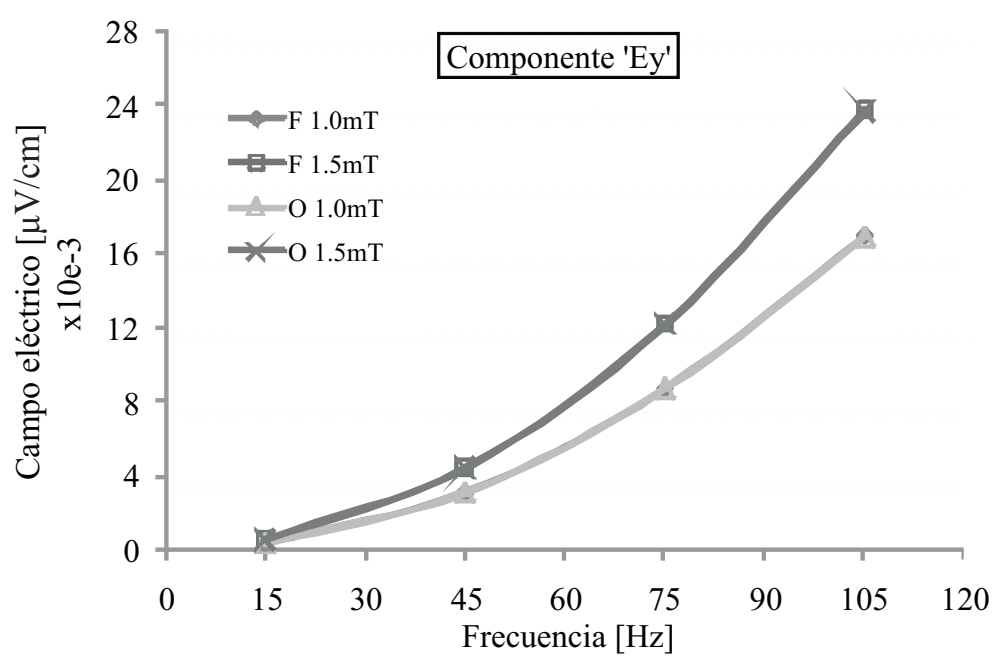

Figura 12. Campo eléctrico inducido vs. Frecuencia componente y, en fibroblastos y osteoblastos a $1.0 \mathrm{mT}$ y $1.5 \mathrm{mT}$.

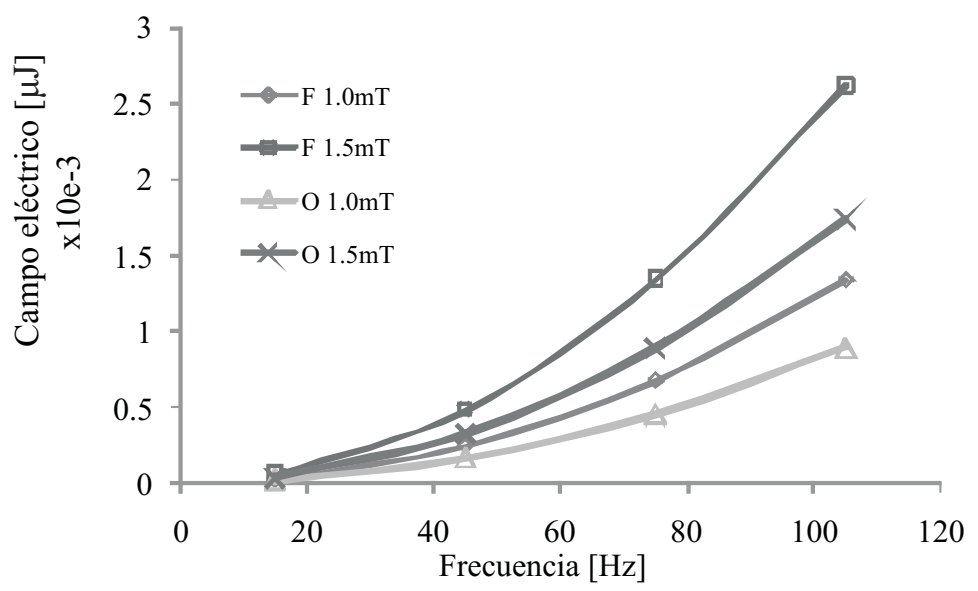

Figura 13. Calentamiento eléctrico inducido vs. Frecuencia, en fibroblastos y osteoblastos a 1,0mT y 1,5mT.

La densidad de corriente inducida fue superior para el material de fibroblastos en 1.5 veces la inducida en los osteoblastos, para 1.0 y $1.5 \mathrm{mT}$. Los valores encontrados para fibroblastos a 1.0 $\mathrm{mT}$ fueron superiores a los encontrados para osteoblastos a $1.5 \mathrm{mT}$. El campo eléctrico inducido a $1.0 \mathrm{mT}$ fue el mismo para los materiales fibroblastos y osteoblastos; igual comportamiento se encontró a $1.5 \mathrm{mT}$.

Las magnitudes del calentamiento eléctrico fueron del orden de $10^{-10} \mathrm{~J}$; con valores mayores para el material de fibroblastos en 1.5 veces los valores en los osteoblastos, tanto para $1.0 \mathrm{mT}$ como para $1.5 \mathrm{mT}$.

\section{Conclusiones}

Se realizó el diseño de un modelo computacional 3D representando un arreglo de células expuestas a campos magnéticos de baja frecuencia. De manera específica se trabajó con los materiales de células de fibroblastos y osteoblastos humanos. Con el desarrollo del modelo 3D se logró estimar un comportamiento de variables eléctricas inducidas en un cultivo celular in vitro y su 
variación al modificar las características de la señal de estimulación en magnitud y frecuencia. El modelo permite adecuar el radio de la bobina Helmholtz y modificar las condiciones de la fuente y la carga.

\section{Agradecimientos}

A COLCIENCIAS y al MD Ortopedista Alfredo Martínez R. de la Facultad de Salud, sección ortopedia de la Universidad del Valle.

\section{Referencias bibliográficas}

Bjordal, JM., Johnson, MI., \& Ljunggreen, AE. (2003). Transcutaneous electrical nerve stimulation (TENS) can reduce postoperative analgesic consumption. A meta-analysis with assessment of optimal treatment parameters for postoperative pain. European Journal of Pain 7, 181-188.

Cromwell, GL., Moneagul, HJ, \& Stanley, TW. (1980). Effect of dietary inclusion of copper sulphate and antibiotics on performance of weaner pigs. Journal of Animal Science, 51, 1347.

Ferguson, M., Byrnes, C., Sun, L., Marti, G., Bonde, P., Duncan, M., \& Harmon, JW. (2005). Wound healing enhancement: electroporation to address a classic problem of military medicine. World Journal of Surgery 29 (Suppl 1), S55-S59.

Fitzsimmons, RJ., Ryaby, JT., Mohan, S., Magee, FP., \& Baylink, DJ. (1995). Combined magnetic fields increase IGF-II in TE-85 human bone cell cultures. Endocrinology 136, 3100-3106.

Fitzsimmons, RJ., Ryaby, JT., Magee, FP., \& Baylink, DJ. (1994). Combined magnetic fields increase net calcium flux in bone cells. Calcified Tissue Internacional 55, 376-380.

Gentzkow, GD. (1993). Electrical stimulation to heal dermal wounds. Journal of Dermatology Surgery \& Oncology 19; 753-758.
Gothelf, A., Mir, LM., \& Gehl, J. (2003). Electrochemotherapy: results of cancer treatment using enhanced delivery of bleomycin by electroporation. Cancer Treatement Review 29, 371-387.

Katsir, G., Baram, SC., \& Parola, AH. (1998). Effect of sinusoidally varying magnetic fields on cell proliferation and adenosine deaminase specific activity. Bioelectromagnetics 19, 46-52.

Lee, RC., Canaday, DJ., \& Doong, H. (1993). A review of the biophysical basis for the clinical application of electric fields in soft-tissue repair. Journal of Burn Care \& Rehabilitation 14, 319-335

Moncada, ME., \& Cadavid, H. (2007). Estimación de variables eléctricas en un muslo 3D con fractura de diáfisis femoral estimulado magnéticamente. Revista Facultad de Ingeniería Universidad de Antioquia 42, 120-131.

Moncada, ME., Martínez, A., \& Pinedo, CR. (2008). Desarrollo experimental y computacional para estimar variables eléctricas inducidas en muestras de fémur bovino estimuladas por campos magnéticos de baja frecuencia. Revista Cubana de Investigaciones Biomédicas, 27 (2), 1-9.

Ojingwa, JC. \& Isseroff, RR. (2003). Electrical stimulation of wound healing. Journal of Investigative Dermatology 121, 1-12.

Rushton, DN. (2002). Electrical stimulation in the treatment of pain. Disability and Rehabilitation $24,407-415$.

Sluka, KA., \& Walsh, D. (2003). Transcutaneous electrical nerve stimulation: basic science mechanisms and clinical effectiveness. Journal of Pain 4, 109-121. 